\title{
Synovitis with non-specific histological changes in synovium in chronic sarcoidosis
}

\author{
DAVID G. PALMER AND H. RALPH SCHUMACHER \\ From the Arthritis-Immunology Center, Veterans Administration Medical Center, the University of \\ Pennsylvania School of Medicine, Philadelphia, PA, and the University of Otago Medical School, Dunedin, \\ New Zealand
}

SUMMARY Polyarthritic episodes in seven patients during the course of chronic sarcoidosis involved the knees and less frequently other joints. Synovial effusions were non-inflammatory or mildly inflammatory. Needle synovial biopsies 10 days to seven weeks after the onset of joint symptoms have revealed varying patterns, including mild lining cell proliferation, occasional vascular congestion, diffuse infiltrates with lymphocytes and histiocytes, but no granulomas. These studies show distinctly less inflammation than in rheumatoid arthritis. Granulomas need not be identifiable in synovium in all chronic sarcoidosis with arthritis.

Key words: synovial fluid, electron microscopy, granulomas, synovial biopsy.

Although an arthritis is a common clinical feature of acute sarcoidosis (Lofgren's syndrome) ${ }^{12}$ it is less frequently recognised during the course of chronic disease. ${ }^{3}$ When an arthropathy does appear under the latter circumstances, a problem in differential diagnosis may arise if the nature of the underlying disorder has not been established, or if the possibility of some coincidental arthritis has to be entertained. The arthritis which may accompany chronic sarcoid arthritis may be acute and evanescent, intermittent with relapses and remissions, or progressive leading to joint destruction. ${ }^{3-5}$ Differentiation from rheumatoid arthritis may not always be easy, because systemic manifestations such as weight loss, tiredness, and sweating are common to both, rheumatoid factor accompanies chronic sarcoidosis in up to $38 \%$ of patients, ${ }^{6}$ and both disorders may be accompanied by subcutaneous nodules. ${ }^{7}$ Further diagnostic confusion with other causes of arthritis can occur as antinuclear antibodies, 8 hyperuricaemia, ${ }^{34}$ and hypercalcae$\mathrm{mia}^{910}$ have all been reported in some patients with sarcoidosis.

Accepted for publication 11 June 1984.

Correspondence to $\mathrm{H}$. Ralph Schumacher, MD, Professor of Medicine, University of PA School of Medicine, Director, Arthritis-Immunology Center, Veterans Administration Medical Center, University and Woodland Avenues, Philadelphia, PA 19104, USA.
Since Sokoloff and Bunim described noncaseating granulomas in three of five surgical biopsy specimens of synovium in cases of chronic sarcoidosis $^{11}$ it has been widely accepted ${ }^{12} 13$ that such changes would probably be found if involved synovium were to be examined histologically. We have reviewed synovial fluid analyses and the light and electron microscopic appearances of synovium obtained by needle biopsy from knee joints developing signs of synovitis during the course of sarcoidosis and have found only non-specific changes in the seven patients studied.

\section{Patients and methods}

All patients (Table 1) had a well established diagnosis of chronic sarcoidosis based on histological evidence of typical non-caseating granulomatous lymphadenitis associated with a compatible clinical picture. With the exception of concurrent systemic corticosteroid therapy, specimens from patients with any additional problems which might have modified the histological features were excluded from the survey. Synovial biopsy was performed with a Parker-Pearson needle, specimens were fixed in buffered formalin, and sections stained with haematoxylin and eosin for light microscopy. For transmission electron microscopy specimens from patients 1 , 
Synovitis with non-specific histological changes in synovium in chronic sarcoidosis 779

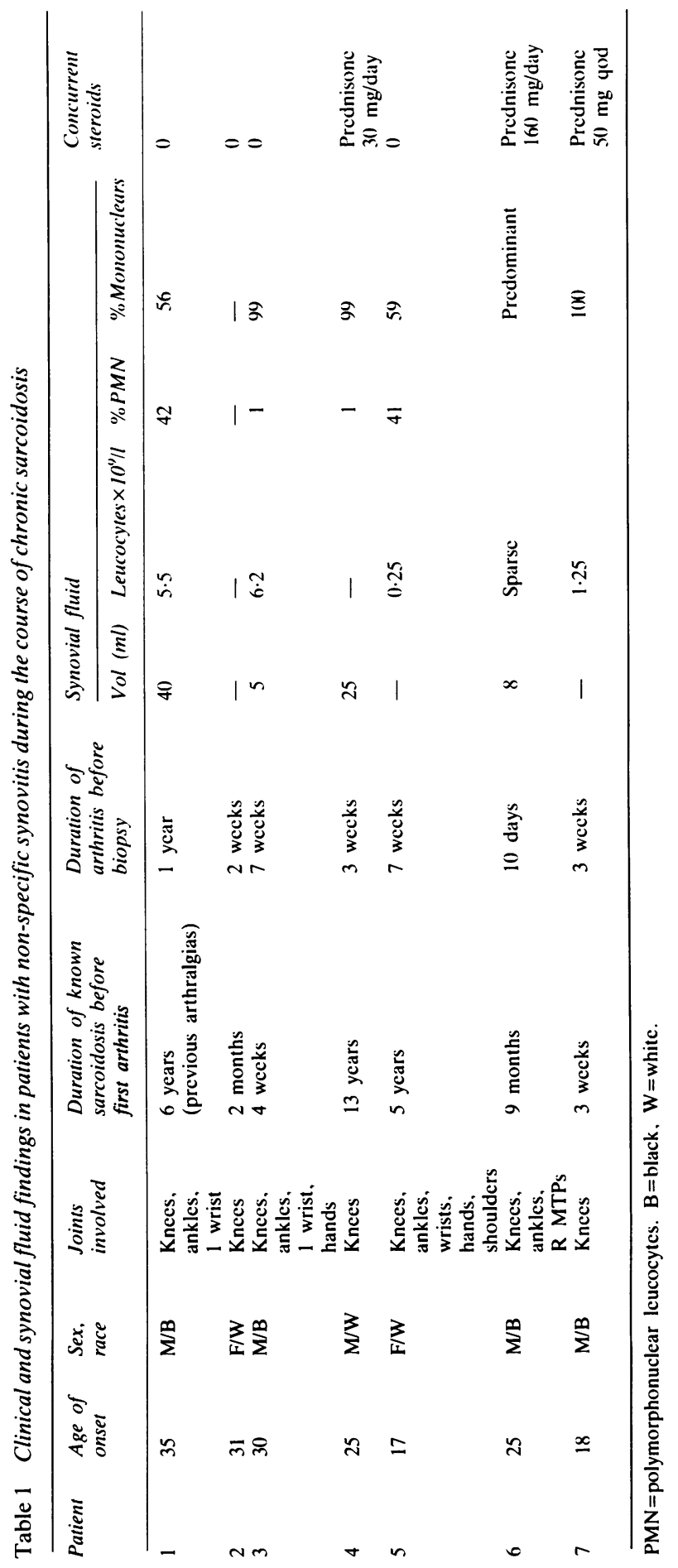


$2,3,4$, and 7 were fixed in 1/2 strength Karnovsky's paraformaldehyde-glutaraldehyde, and processed as previously described. ${ }^{14}$ The gross appearances of knee synovial fluid aspirates, leucocyte counts, and cell differentials on Wright's stained smears were recorded. All fluids were examined with compensated polarised light and no crystals were found.

\section{Results}

CLINICAL PRESENTATION (Table 1)

Four of the seven patients had initially presented with constitutional symptoms including anorexia, weight loss, or fever. All had pulmonary involvement, but this varied from asymptomatic hilar lymphadenopathy (without erythema nodosum) to severe respiratory impairment with productive cough and shortness of breath. Two patients (cases 3 and 7) had biopsy proved hepatic granulomas and patient 4 had hepatomegaly. These three had splenomegaly. Case 3 had conjunctival granulomas and case 7 had radiological evidence of bone involvement. Compatible skin lesions were present in five patients with biopsy proof of granulomas in patients 1 and 3 .

All patients had at least two joints involved. Lower limb joint involvement, particularly of the knees, predominated and was usually symmetrical. Upper limb joint involvement was less common and tended to be asymmetrical. Morning stiffness was only an occasional minor complaint. Arthritis had persisted as long as one year before examination, though most patients were seen during the first 2-7 weeks of the arthritis.

The interval between the appearance of symptoms attributed to the onset of sarcoidosis and initial joint manifestations varied from three weeks to 13 years. The long-term course of the arthritis in most of our patients is not known, as many of them have had limited follow-up visits. Patient 1 has had recurrent non-destructive arthritis over 3 years. No evidence of articular damage was detected in radiographs of the involved joints in any patient.

All synovial fluid samples had non-inflammatory or mildly inflammatory characteristics, being relatively clear, colourless or straw-coloured, and of good viscosity. The total white cell count ranged from 0.25 to $2 \cdot 65 \times 10^{9} / 1$ with $56-100 \%$ mononuclear cells. There were $9-82 \%$ lymphocytes and many cells that appeared to be synovial lining cells or other large mononuclear cells.

\section{LIGHT MICROS COPY}

The histological appearances were heterogeneous.

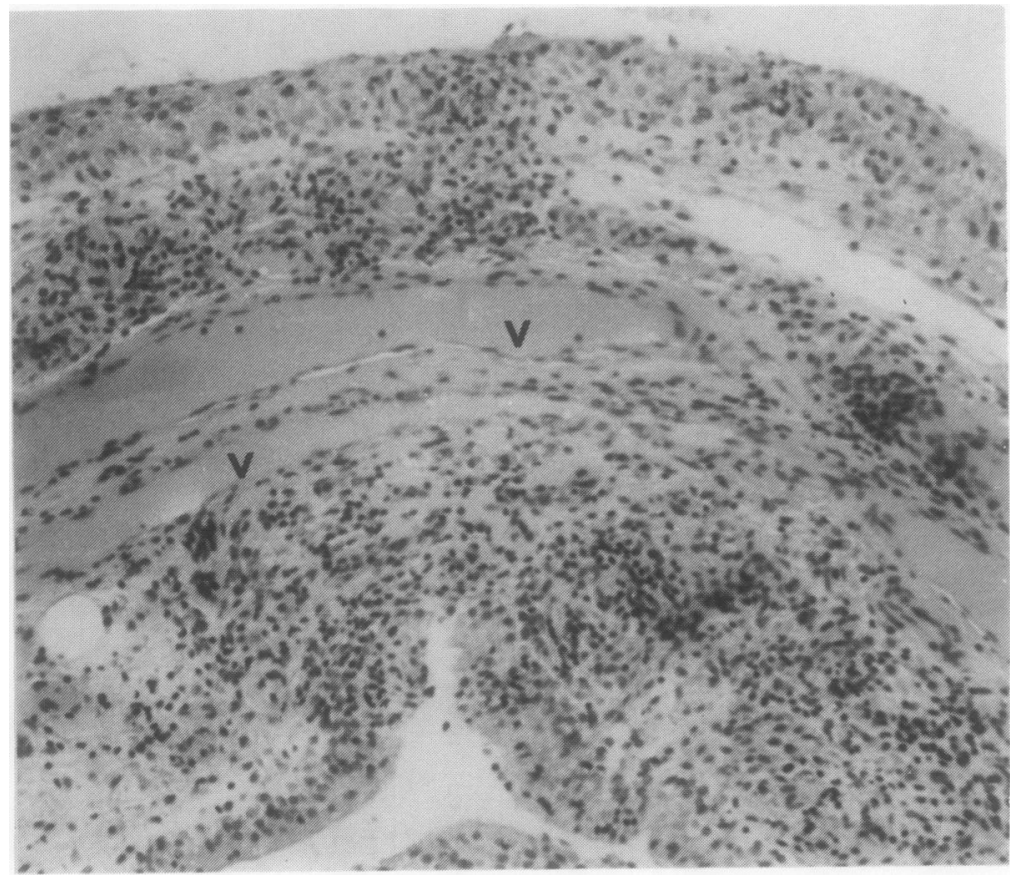

Fig. 1 Patient 1. Hyperplasia and hypertrophy of the synovial lining layer (at top and bottom) is apparent. There is a diffuse infiltrate of tissue histiocytes and lymphocytes. Vessels (V) are congested. ( $H$ and $E, \times 30)$. 
Fibrin deposition on the synovial lining ceils was usually present but was inconspicuous and discontinuous. Synovial hypertrophy and hyperplasia were likewise variable, being absent in some specimens but forming a mantle some $5-7$ cells deep in others (Fig. 1). The size of specimen available did not allow for an adequate evaluation of villous hypertrophy, but some increased complexity of these structures was seen.

There was a rather diffuse increase in interstitial histiocytes and lymphocytes (Figs. 1, 2) and although perivascular localisation was encountered compact foci of cells were not seen. Diffuse infiltration was best seen in patients 1,2, and 7. Closely packed epithelioid cells or multinucleated cells were not found (Figs. 1, 2). Plasma cells were relatively sparse, and polymorphonuclear leucocytes were usually seen only in association with debris.

Where histiocytic infiltration was marked, vascularity appeared to be decreased. In other areas small blood vessels were numerous (Figs. 1,2). Perivascular and interstitial fibrosis of marked degree was present in part of the specimen from patient 3 . Necrosis was the dominant finding in specimens from patients 3 and 5 with loss of intimal cells, necrosis of interstitial cells, and hyaline change in the interstitium itself.

\section{ELECTRON MICROSCOPY}

Ultrastructural studies allowed identification of small numbers of synovial lining cells with no unusual features. The majority of infiltrating cells seen seemed to be histiocytes, and in four of the five patients studied by EM these contained considerable finely granular material in their phagocytic vacuoles. Vessels occasionally had multilaminated basement membranes, and there were occasional pale endothelial cells or gaps between them. There was some interstitial necrotic debris. Mast cells were seen to be degranulated in two specimens. Specifically not found were any virus-like or bacterial particles, tubuloreticular structures, or electron dense deposits in vessel walls.

\section{Discussion}

Involvement of joints in the course of acute sarcoidosis and a chronic persistent arthritis with histological evidence of a non-caseating granulomatous reaction accompanying involvement of other organs in chronic disease ${ }^{19}$ are closely identified with these two seemingly distinct forms of sarcoid reaction. The duration of the synovitis before biopsy seems not to be entirely critical in determining the presence of granulomas in that these have been



Fig. 2 Patient 7. Synovial blood vessels (V) are numerous. The histiocytic and lymphocytic cellular infiltrate shows little evidence of focal accumulation ( $H$ and $E, \times 60)$. 
recognised by the fifth week. ${ }^{y}$ In contrast, the nature of the mild and often transient arthralgias and arthritides which may also punctuate the course of chronic sarcoidosis have not been well defined. ${ }^{3415}$ Sporadic reports which include two of the five patients reported on by Sokoloff and Bunim ${ }^{11}$ have indicated that the histological changes may be of a non-specific nature. ${ }^{59}$ 13-16

As chronic sarcoidosis may run a course of many years in patients who may be immunologically compromised (both as a result of their disease $\mathrm{e}^{17}$ and through long continued corticosteroid therapy), other types of arthritis such as infective arthritis might well be occasionally encountered. It was our experience in reviewing the case notes of all patients with sarcoidosis and arthritis that documented unrelated disorders which might have contributed to the synovial pathology were in fact common. Such cases were excluded from this study. The earlier diagnosis and treatment of the arthritis as rheumatoid arthritis, the diagnosis and treatment of the illness at its onset as tuberculosis, a history of non-specific urethritis, and the culture of Aspergillus fumigatus from a granulomatous node in patients with otherwise typical sarcoid disease were among associated problems encountered. Although it was not practical to exclude patients treated with corticosteroid therapy from this series, the possibility of corticosteroid induced osteonecrosis was considered in the differential diagnosis. ${ }^{18}$

Our principal conclusions are that a non-caseating granulomatous reaction is not necessarily a readily detectable histological feature of the episodic polyarthritis which may evolve in the course of chronic systemic sarcoidosis, and that synovial fluid from the involved joints is usually of a non- or mildly inflammatory type even though the onset of the arthropathy may be acute and dramatic. The preponderance of mononuclear cells in the synovial fluid is similar to the findings in most previous reports. ${ }^{11} 13^{19}$ The diffuse synovial infiltrate consisting principally of histiocytic cells and lymphocytes might be representative of the change preceding epithelioid transformation and granuloma formation $^{20}$ or it might be due to other mechanisms in these patients. Although some inflammatory cell infiltration was common, it was less marked than that seen in typical chronic rheumatoid disease, so that adequate biopsies might help separate this from sarcoidosis even in the absence of granulomas.

Electron microscopy provided only very nonspecific findings and did not yield any positive evidence for mechanisms that might be involved in this type of arthritis. Whether any of the interstitial or phagocytosed granular material might contain antigenic material important in pathogenesis would require immunomorphological studies that were not done on these specimens.

While better surgical biopsy specimens might possibly have yielded tissue containing focal granulomas in some patients, it is doubtful if this procedure can ordinarily be justified in patients with a diagnosis of sarcoidosis alreadly well substantiated, so that most clinical evaluations of arthritis in sarcoidosis may well depend on interpretation of needle biopsies as used here. Study of additional patients in which long-term follow-up can be obtained may clarify whether or how often this mild synovitis progresses to a destructive granulomatous arthritis.

\section{References}

1 Lofgren S. Primary pulmonary sarcoidosis. 1. Early signs and symptoms. Acta Med Scand 1977; 145: 424-31.

2 Kitridou R P, Schumacher H R. Arthritis of acute sarcoidosis. Arthritis Rheum 1970; 13: 328 (abstr).

3 Gumpel J M, Johns C J, Shulman L E. The joint disease of sarcoidosis. Ann Rheum Dis 1967; 26: 194-205.

4 Spilberg I, Siltzbach L E, McEwen C. The arthritis of sarcoidosis. Arthritis Rheum 1969; 12: 126-37.

5 LeGoff P, Jaffres R, Schwarzberg C, Brousse A, LeRoy J P. Arthrite chronique destructrice du genou d'origine sarcoidosique associéc à des géodes des os longs. Rev Rhum Mal Osteoartic 1982; 49: 647-52.

6 Oreskes I, Siltzbach L E. Changes in rheumatoid factor activity during of sarcoidosis. Am J Med 1968; 44: 60-7.

7 Boyd R E, Andrews B S. Sarcoidosis presenting as cutaneous ulceration, subcutaneous nodules and chronic arthritis. $J$ Rheumatol 1981; 8: 311-36.

8 Veien N K, Hardt F, Bendixen G, et al. Humoral and cellular immunity in sarcoidosis. Acta Med Scand 1978; 203: 321-6.

9 Bianchi F A, Keech M K. Sarcoidosis with arthritis. Ann Rheum Dis 1964; 23: 463-79.

10 David N J, Verner J V, Engel F L. The diagnostic spectrum of hypercalcemia. Am J Med 1962; 33: 88-110.

11 Sokoloff L, Bunim J J. Clinical and pathological studies of joint involvement in sarcoidosis. N Engl J Med 1959; 260: 842-7.

12 Arnold W J. The rheumatic manifestations of sarcoidosis. In: Kelley W N, ed. Textbook of rheumatology. Philadelphia: Saunders, 1981: 1531-7.

13 Schumacher H R. Sarcoidosis. In: McCarty D J, ed. Arthritis and allied conditions. 9th ed. Philadelphia: Lea and Febiger. 1979: 918-24.

14 Schumacher H R, Newton C, Halliwell R E W. Synovial pathologic changes in spontaneous canine rheumatoid-like arthritis. Arthritis Rheum 1980; 23: 412-23.

15 Kaplan $H$. Sarcoid arthritis with a response to colchicinc. $N$ Engl J Med 1960; 263: 778-81.

16 Ferguson R H, Paris J. Sarcoidosis. Study of twenty-nine cases with a review of splenic, hepatic, mucous-membrane, retinal, and joint manifestations. Arch Intern Med 1958; 101: 1065-84.

17 Daniele R P, Dauber J H, Rossman M D. Immunologic abnormalities in sarcoidosis. Ann Intern Med 1980; 92: 406-16.

18 Heimann W G. Freiberger R H. Avascular necrosis of the femoral and humeral heads after high-pose corticosteroid therapy. N Engl J Med 1960; 263: 672-5.

19 Varkey B. Synovial fluid in sarcoidosis. Ann Intern Med 1974; 81: 557.

20 Crystal R G, Roberts W C, Hunninghake G W, Gadek J E, Fulmer J D, Line B R. Pulmonary sarcoidosis: a disease characterized and perpetuated by activated lung $T$ lymphocytes. Ann Intern Med 1981; 94: 73-94. 